Supporting Information

\title{
Integument of Cytoplasmic Membrane onto Cationic DNA Condensates for Selective Gene Expression at Homologous Cells
}

Hongyan Cui ${ }^{\mathrm{ab}}$, Jing Xia ${ }^{\mathrm{b}}$, Liuwei Zhang ${ }^{\mathrm{b}}$, Ming Qian ${ }^{\mathrm{b}}$, Hao Wang ${ }^{\mathrm{b}}$, Jingyun Wang*ab, Qixian Chen*ab

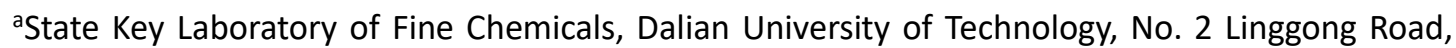
Dalian 116024, China

bSchool of Bioengineering, Dalian University of Technology, No. 2 Linggong Road, Dalian 116024, China.

*E-mail: wangjingyun67@dlut.edu.cn.

*E-mail: qixian@dlut.edu.cn. 
S1. Characterization of fluorescent CQD and CQD-OEI.
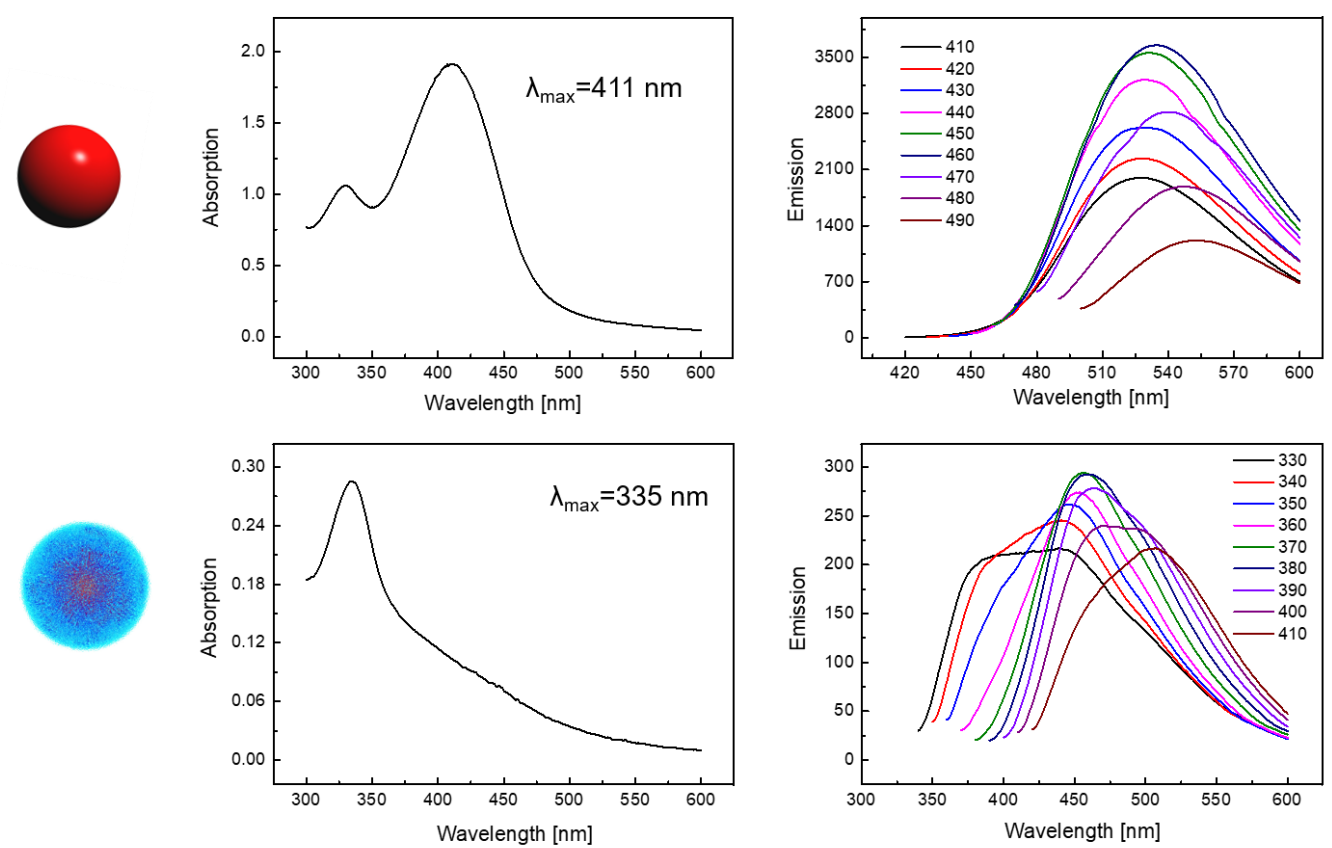

Fig. S1 Absorption and fluorescence emission spectra of the yielded luminogens of CQD and CQDOEI.

\section{S2. Electrostatic complexation behaviors of CQD-OEI/pDNA.}

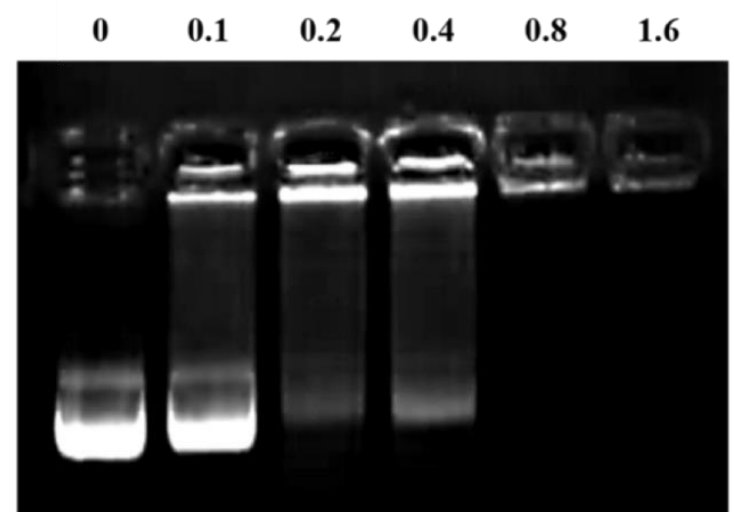

Fig. S2 Gel retardation assay to characterize the complexation behaviors of CQD-OEI and pDNA at varied weight ratios.

S3. Cell viabilities of CQDs-OEI/M. 

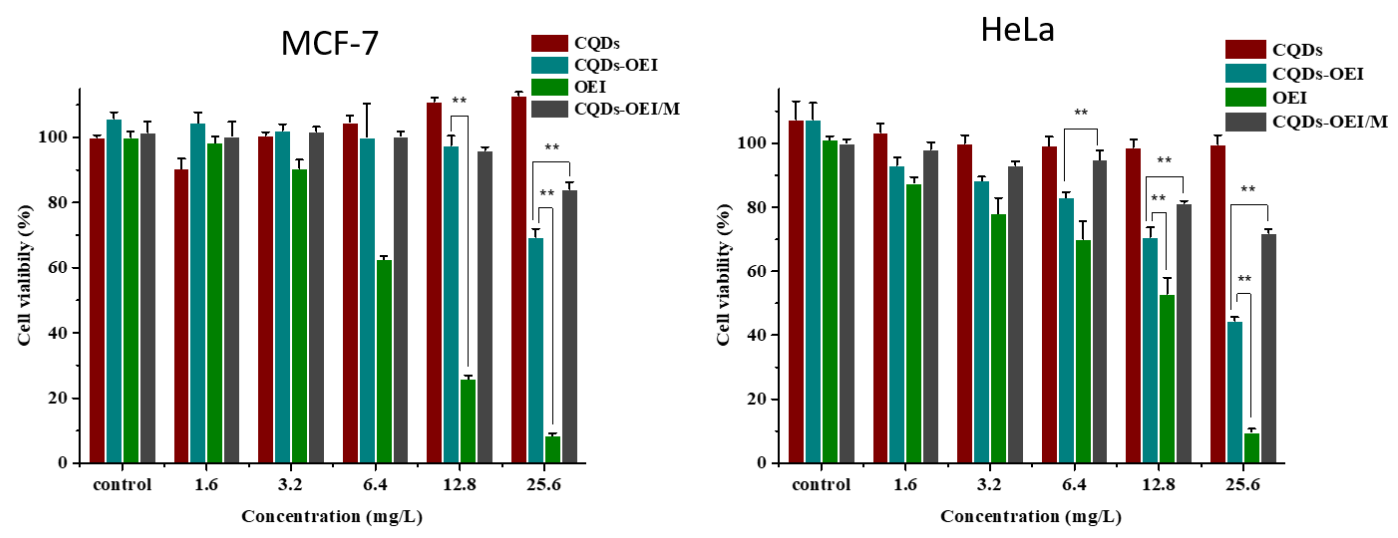

Fig. S3 Cell viabilities of CQDs-OEI post cytoplasmic membrane integuments from MCF-7 and HeLa cells under incubation with the homologous cells.

S4. Cellular uptake efficiencies of CQD-OEI/M(Hep-G2) in Hep-G2 and COS-7 cells.

(a)

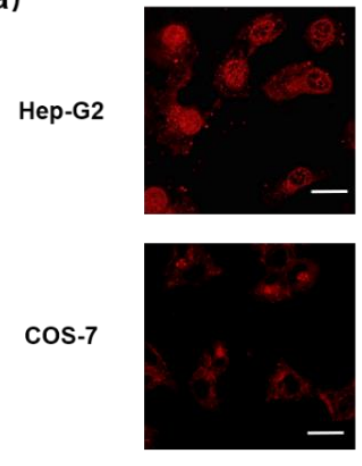

(b)

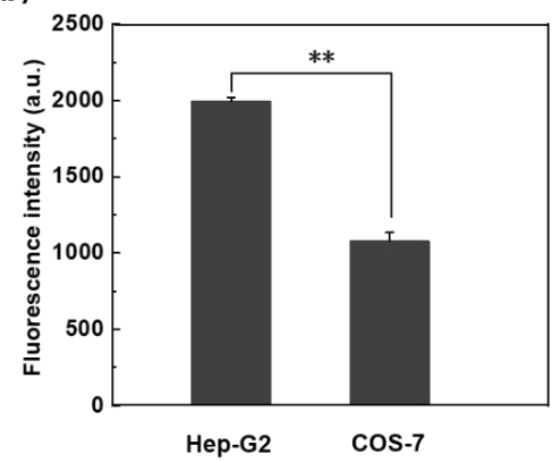

Fig. S4 Cellular uptake efficiencies of CQD-OEI/M(Hep-G2) in homologous cancerous Hep-G2 cells and heterologous normal COS-7 cells. (a) CLSM measurements were conducted at $8 \mathrm{~h}$ post incubation (red: fluorescence from CQD, scale bar: $20 \mu \mathrm{m}$ ); (b) The quantified data were summarized into the right bar graph (Student $t$-test, ${ }^{* *} p<0.01$ ).

S5. Gene expression efficiencies of gene delivery constructs OEI/pDNA/M(Hep-G2) in homologous and heterologous cells. 

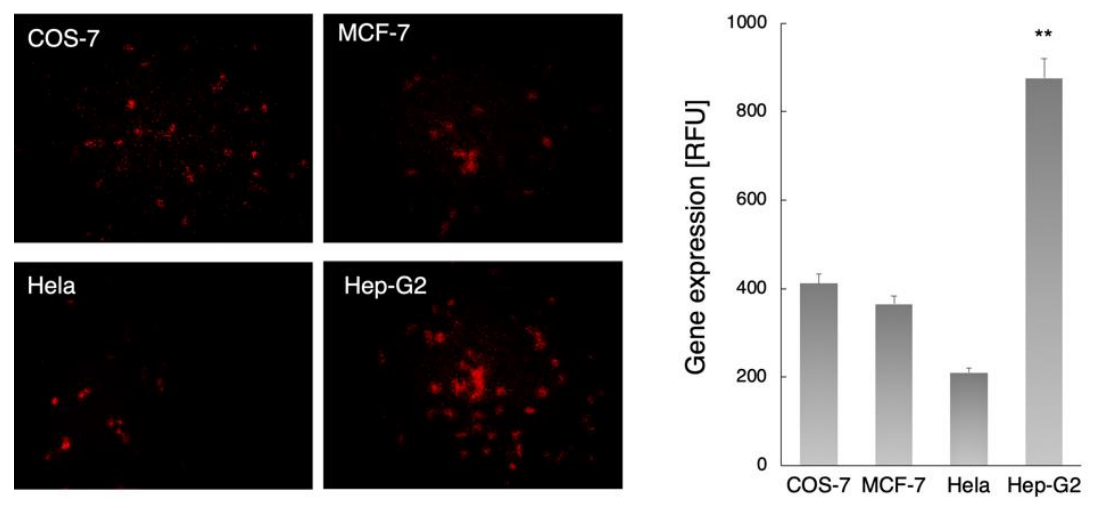

Fig. S5 Gene expression efficiencies of OEI/pDNA/M(Hep-G2) in homologous and heterologous cells. CLSM measurements were conducted at $48 \mathrm{~h}$ post incubation [red: expression of pDNA encoding red fluorescence protein (RFP)], and the quantified data were summarized into the right bar graph (Student $t$-test, ${ }^{* *} p<0.01$ ). 